\title{
Dissociable Effects of Natural Image Structure and Color on LFP and Spiking Activity in the Lateral Prefrontal Cortex and Extrastriate Visual Area V4
}

\author{
Stefanie Liebe, ${ }^{1}$ Nikos K. Logothetis, ${ }^{1,2}$ and Gregor Rainer ${ }^{1,3}$ \\ ${ }^{1}$ Department of Physiology of Cognitive Processes, Max Planck Institute for Biological Cybernetics, 72076 Tuebingen, Germany, ${ }^{2}$ Faculty of Medical and \\ Human Sciences, The University of Manchester, Manchester M13 9PT, United Kingdom, and 32Department of Medicine, University of Fribourg, CH-1700, \\ Switzerland
}

Visual perception is mediated by unique contributions of the numerous brain regions that constitute the visual system. We performed simultaneous recordings of local field potentials (LFPs) and single unit activity (SUA) in areas V4 and lateral prefrontal cortex to characterize their contribution to visual processing. Here, we trained monkeys to identify natural images at different degradation levels in a visual recognition task. We parametrically varied color and structural information of natural images while the animals were performing the task. We show that the visual-evoked potential (VEP) of the LFP in V4 is highly sensitive to color, whereas the VEP in prefrontal cortex predominantly depends on image structure. When examining the relationship between VEP and SUA, we found that stimulus sensitivity for SUA was well predicted by the VEP in PF cortex but not in V4. Our results first reveal a functional specialization in both areas at the level of the LFP and further suggest that the degree to which mesoscopic signals, such as the VEP, are representative of the underlying SUA neural processing may be brain region specific within the context of visual recognition.

\section{Introduction}

In humans, the study of evoked electric potentials with electroencephalography has led to a wealth of knowledge about the processing of sensory stimulus events (Hillyard, 1993). In nonhuman primates, however, most of what is known about neural correlates of visual perception comes from recording spiking activity in single neurons (Hubel and Wiesel, 1968; Zeki, 1973; Desimone et al., 1984; Maunsell and Newsome, 1987). To understand the relationship between these brain signals, an increasing number of studies have started to compare sensory characteristics of spiking activity and invasive electric local field potentials (LFPs) obtained from extracellular multielectrode recordings in nonhuman primates.

The comparison of visual sensitivity of both types of signals yielded mixed results. Several studies demonstrated a close correspondence in sensory properties between spiking activity and evoked responses of mesoscopic brain signals, i.e., neural activity at

\footnotetext{
Received April 8, 2010; revised April 1, 2011; accepted April 14, 2011.

Author contributions: S.L., N.K.L., and G.R. designed research; S.L. and G.R. performed research; S.L. analyzed data; S.L., N.K.L., and G.R. wrote the paper.

This work was supported by the Deutsche Forschungsgemeinschaft Sonderforschungsbereich 550, a European Science Foundation European Young Investigator award to G.R., and by the Max Planck Society. We thank B. Averbeck, I Monosov,K. Thompson, and S. Zeki for helpful comments on this manuscript and Andreas Bartels and Jakob Macke for helpful discussions throughout the project.

Correspondence should be addressed to either of the following: Stefanie Liebe at her present address; or Gregor Rainer, Department of Physiology of Cognitive Processes, Max Planck Institute for Biological Cybernetics, Spemannstrasse 38, 72076 Tuebingen, Germany, E-mail: sliebe@tuebingen.mpg.de or gregor.ranier@unifr.ch.

S. Liebe's present address: Department of Neuroscience, Physiology and Pharmacology, University College London, 21 University Street, WC1E 6DE London, United Kingdom. E-mail: s.liebe@ucl.ac.uk.

DOI:10.1523/JNEUROSCI.1791-10.2011

Copyright $\odot 2011$ the authors $\quad 0270-6474 / 11 / 3110215-13 \$ 15.00 / 0$
}

larger spatial scales (Victor et al., 1994; Liu and Newsome, 2006; Katzner et al., 2009). This has been taken as evidence for the hypothesis that stimulus-evoked components of LFPs reflect the local average of the underlying spiking population response. In contrast, other studies have shown that stimulus-evoked LFPs carry different information from that contained in spiking activity (Kreiman et al., 2006; Nielsen et al., 2006; Asher et al., 2007; Monosov et al., 2008). For example, a recent study reported a strong correspondence between sensory characteristics of LFPs within a given area and its presumed feedforward input (Khawaja et al., 2009). These findings support the hypothesis that LFPs reflect sensory characteristics of the input into an area, and that this input exhibits different stimulus selectivity than neurons contributing to local spiking.

The discrepancy in results suggests that there is no simple mapping between sensory selectivity of spiking activity and LFPs and highlights the importance of studying both signals to elucidate their respective role in visual perception (Rainer, 2008).

The interpretation of the relationship between both signals is further complicated by the fact that most of the studies were not only using different experimental paradigms but were also conducted in different cortical regions. Whether and how sensory characteristics of the two signals are related could thus depend on factors unique to a given area. However, this question can only be addressed by simultaneously recording neural activity in multiple regions using identical experimental and stimulus conditions.

In this study, we therefore sought to characterize the relationship between visual characteristics of spiking activity and LFPs in two cortical regions that play prominent and distinct roles in visual object processing. Specifically, we investigated visual responses of spiking activity and LFPs to natural image manipula- 
tions in the extrastriate area V4 and the lateral prefrontal cortex (IPF). Despite the large amount of literature describing visual characteristics of single neurons in V4 and IPF, the question of how cortical LFPs encode sensory information about objects compared with spiking activity in these regions has remained elusive. In addition, by using identical stimulus conditions we were able to directly compare the relationship between sensory properties of spiking activity and LFPs between both regions.

\section{Materials and Methods}

Behavioral task and subjects. Two adult male monkeys (Macaca mulatta) participated in the experiments. All studies were approved by the local authorities (Regierungspräsidium, Tübingen, Germany) and were in full compliance with the guidelines of the European Community for the care and use of laboratory animals (European Union Directive 86/609/EEC). A description of the behavioral paradigm and stimuli used is given in a previous study (Liebe et al., 2009) and also in the supplemental material. In total, a set of seven different randomly selected natural images was chosen from the Corel-Photo-CD "Corel Professional Photos" comprising a collection of natural images showing birds, flowers, monkeys, and butterflies in their natural surroundings. All images were familiar to the animals. For each experiment and animal, the same set of images was presented, and we did not select images based on selectivity of neuronal responses of either V4 or prefrontal neurons.

The behavioral task of the monkeys was a delayed matching to sample task. The monkeys initiated a trial-start by grasping a lever and fixating on a small fixation spot on the center of the screen. After successful fixation and grasping for $1000 \mathrm{~ms}$, a first stimulus appeared on the screen for $250 \mathrm{~ms}$, the so-called sample stimulus. The sample stimulus was presented in any of the 12 stimulus conditions shown in Figure $1 B$. The sample stimulus was followed by a delay period of $1500 \mathrm{~ms}$ during which the monkey holds fixation. After the delay, a second stimulus, the socalled test stimulus is presented. The test stimulus could be any of the non-degraded (100\% coherence) images. For each color condition the corresponding test stimulus is shown in Figure $1 \mathrm{~A}$ on the left-most column. The monkeys were rewarded for a lever release, whenever the test stimulus matched the sample stimulus, i.e., if the sample was either identical to the test stimulus or a degraded version ("match"). Whenever the test stimulus did not match the sample ("nonmatch"), the monkeys' task was to withhold the lever release until, after a brief delay of $200 \mathrm{~ms}$, a second test stimulus appeared that always matched the sample. This procedure ensured that the monkey had to initiate a behavioral response on every trial. The monkeys were rewarded with juice for every correct trial, and randomly for the conditions in which the sample stimulus was a pure noise stimulus (i.e., $0 \%$ coherence). In each experiment, $50 \%$ of the trials were "match"-trials and $50 \%$ were "nonmatch"-trials, so that on pure noise-trials monkeys could perform at maximally $50 \%$ correct on average. Within one session, the different trial types were randomly interleaved. The monkeys completed at least 10 repetitions for each condition during a recording session.

To assess behavioral performance, we first calculated psychophysical performance (\% correct responses) at each degradation level for each monkey individually per recording session and subsequently averaged the performance across sessions ( $N=10$ and 15 for monkey 1 and 2$)$. We subsequently fitted a psychometric function (logistic function fit) using the psignifit toolbox version 2.5.6 for Matlab (Wichmann and Hill, 2001a,b).

Electrophysiology. A detailed description of the surgical procedures and recording apparatus can be found in the supplemental material. All data analyses were performed using Matlab (MathWorks). To obtain visualevoked responses, we first downsampled the local field potential to 1000 $\mathrm{Hz}$ and low-pass filtered it with a second-order Butterworth filter at a cutoff frequency of $25 \mathrm{~Hz}$. Trials in which responses that contained voltages larger than 3.5 SDs of the average response or larger than 3.5 SDs of the variability across the entire trial period were discarded as outliers (on average for both monkeys and areas $8 \%$ of trials). These large variations in voltage resulted from artifacts due to movements of the animal or the recording apparatus; the procedure was confirmed by visual inspection of the discarded trial activity.

Analysis of visual-evoked potentials. We analyzed the resulting evoked activity in the time period of $200 \mathrm{~ms}$ preceding and $800 \mathrm{~ms}$ following the onset of the visual stimulus (sample stimulus). Visual-evoked potentials were calculated by stimulus-locked averaging of the local field potential (LFP) data across the trials. Within the manuscript we refer to the evoked visual response of the local field potential as visual-evoked potential (VEP) and the underlying recorded signal as LFP in a more general sense.

For the analyses involving single LFP sites, we analyzed the activity of 32 sites and 78 sites in V4 of monkey 1 and 2, respectively. In PF, we analyzed the activity of 41 and 66 sites for monkey 1 and 2, respectively. An LFP site was determined visually responsive if its average response during the presentation of the visual stimulus differed significantly from the average baseline activity ( $p<0.01$, paired $t$ test across trials). We defined the baseline period as the activity during the $200 \mathrm{~ms}$ before stimulus onset. To obtain VEP amplitudes, we first assessed the time of the maximum-negative peak deflection for each site and trial within a poststimulus onset window of $100-300 \mathrm{~ms}$ and subsequently averaged the activity within a $50 \mathrm{~ms}$ window surrounding the peak. Subsequently, we converted the negative amplitude values of the VEP into positive values by multiplying with -1 . Amplitudes across different stimulus conditions were normalized by the maximum value observed across the mean of the different color conditions for each coherence level. We also evaluated the latencies of the peak responses between stimulus conditions. We defined the peak latency as the time (ms) from sample onset at which the most negative deflection occurred during the visual response window (100-300 ms poststimulus onset). The latency was assessed on a single trial basis. Across the different conditions, we did not find any significant differences regarding the peak latencies for either of the monkeys (two-way nonparametric ANOVA, V4: factor 1 "color condition," factor 2 "coherence" and interaction, $p>0.05$; PF: factors 1,2 and interaction, $p>0.05$ ). Between areas both monkeys showed higher peak latencies for PF cortex compared with V4, although the comparison only reached statistical significance for one animal and was at trend level for the other animal (monkey 1 mean peak latency V4 $199 \mathrm{~ms}, \mathrm{SD}=12.1, \mathrm{PF}$ $207.1, \mathrm{SD}=20.8$, independent $t$ test, $p>0.05$; monkey 2 peak latency V4 $174 \mathrm{~ms}, \mathrm{SD}=17, \mathrm{PF} 188.7, \mathrm{SD}=16.7, p<0.001$ ).

Slope estimation for comparing sensitivity to color and noise from individual sites. On the average across LFP sites we found a monotonic relationship between amplitude versus coherence functions in V4 as well as in PF. We wanted to describe the nature of this relationship also at the level of individual sites and assess its statistical significance. As we have only four stimulus levels, we estimated slope parameters for individual sites by performing linear regressions on single-trial amplitude versus coherence functions for each color condition separately. To estimate the goodness of fit of our linear regressions, we examined the determination coefficient $\left(R^{2}\right)$. In V4, $R^{2}$ estimates for the achromatic condition were not significant in both monkeys ( $p>0.05$ ), while $R^{2}$ values were significant/at trend level in the colored stimulus conditions $(p<0.05$ for monkey 1 and $p=0.08$ for monkey 2 ), indicating a good linear fit to the data in these conditions. For PF in both monkeys, all $R^{2}$ values were significant $(p<0.001)$, also indicating good linear fits to prefrontal data.

Correlation analysis. For the combined analysis of LFP and single unit activity, only pairs of sites were chosen that were recorded at the same electrode. In addition, both single unit activity (SUA) as well as the visual-evoked response had to be classified as visually responsive, i.e., there had to be both a significant increase in spiking activity as well as a significant negative deflection of the evoked response of local field potential at stimulus onset for a given SUA-LFP pair. To identify visually responsive units, the same method as for the LFP sites was used (see above). To estimate single unit activity, we first obtained spike-density functions by convolution of the spike trains with an exponential density function (Monosov et al., 2008), using a length factor $\lambda=25 \mathrm{~ms}$ and a resolution of $1 \mathrm{~ms}$. Then, we averaged spiking activity within the same time window as was used for assessing the LFP peak-amplitudes and normalized the activity in the same way as for VEP responses (see above). In total, we obtained 58 pairs of simultaneously recorded sites of LFP and spiking activity in V4 (18 and 40 sites for monkeys 1 and 2, respectively), 
A

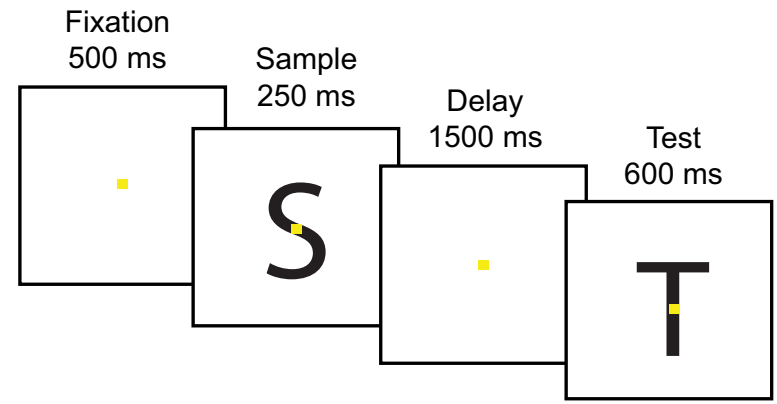

B

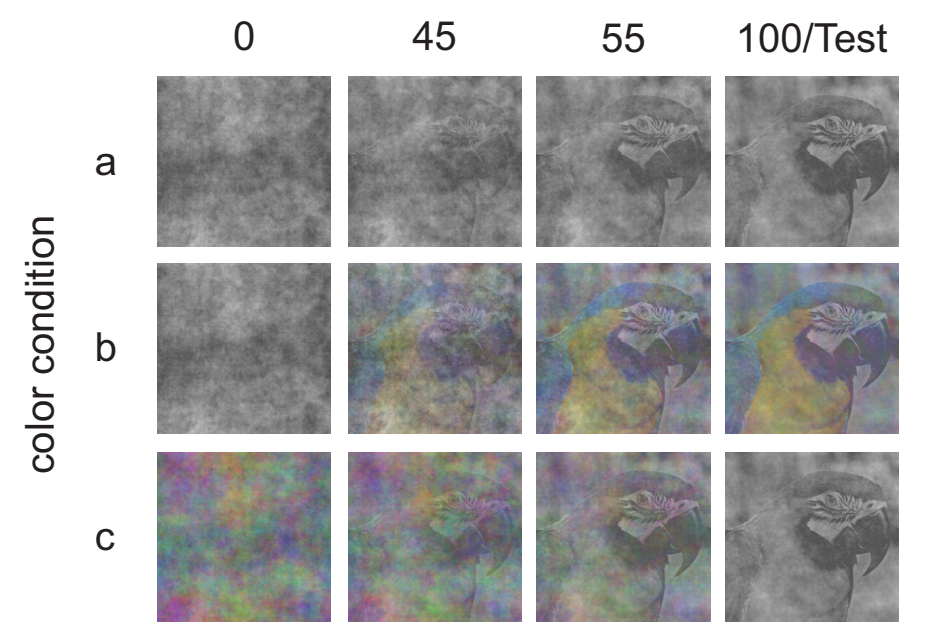

C

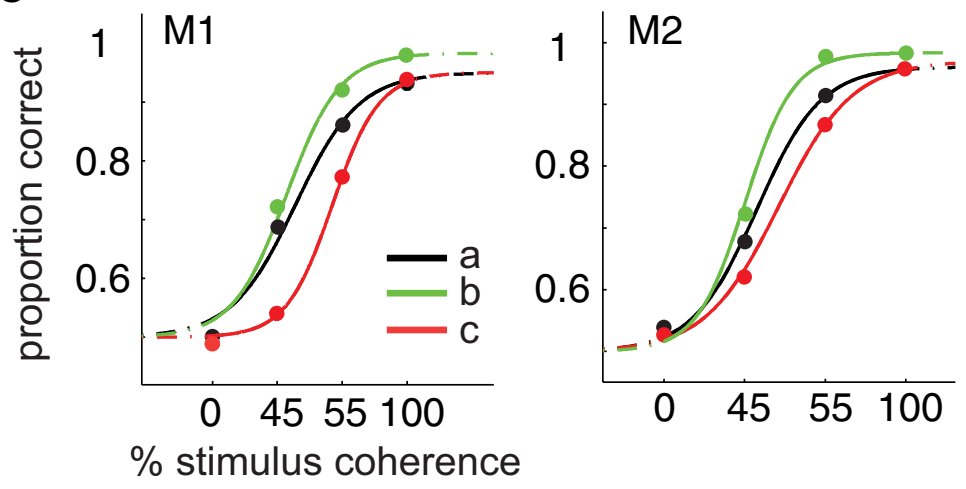

Figure 1. Behavioral task and stimulus conditions for example image. $A$, The sequence of trial events and respective time of events. After fixation, a sample stimulus is presented for $250 \mathrm{~ms}$. This is followed by a delay period of $1500 \mathrm{~ms}$ during which observers hold fixation and no stimulus is shown. After the delay period, a test stimulus is shown. Monkeys were rewarded when releasing the lever in match trials and withholding the response in nonmatch trials. Fifty percent of the trials are match trials, $50 \%$ are nonmatch trials. $\boldsymbol{B}$, Example stimulus shown at various stimulus conditions used in the present experiment. Each row depicts a combination between a noise pattern ( $0 \%$ coherence) and a natural image ( $100 \%$ coherence) at intermediate levels ( $45 \%$ and $55 \%$ coherence). $\boldsymbol{a}$, Achromatic condition; an achromatic noise mask is interpolated with an achromatic natural image. $\boldsymbol{b}$, image color condition; an achromatic noise mask is interpolated with a colored natural image. c, colored noise condition; a chromatic noise mask is interpolated with an achromatic natural image. C, Behavioral recognition performance of both monkeys in DMS task. Each graph plots the proportion of correct responses as a function of stimulus coherence in each color condition $A-C$ for monkey 1 (left) and monkey 2 (right), respectively. Both animals show highest recognition performance in condition $B$, intermediate performance in condition $A$, and worst performance in condition C.

and in PF we analyzed the responses of 51 pairs of simultaneously recorded sites of LFP and spiking activity (32 and 19 sites for monkeys 1 and 2 , respectively) that both showed visual activity. To assess stimulusselectivity similarity between LFP and SUA sites, we computed Spearman's rank-correlation coefficients between single-trial VEP amplitudes and single-trial spiking activity across the $0,45,55$, and $100 \%$ coherence condition. We used the rank correlation rather than the metric correlation because it is more robust to outliers and does not assume a particular relationship (for example strictly linear) other than a monotonic relationship between the two variables under study. The similarity in sensitivity across coherence conditions was assessed for each color condition separately. Before we computed the correlation, we shuffled the trial order per coherence level, such that correlations due to instantaneous similarities in changes of amplitudes and spiking activity were discarded and only correlations due to changes in coherence levels remained. Furthermore, to account for variability in latencies of amplitude and spiking activity, we computed the rank correlation along a time axis ranging from $200 \mathrm{~ms}$ before until $800 \mathrm{~ms}$ after stimulus onset. The correlations were computed with a 1 ms resolution, and subsequently a moving average procedure was applied encompassing 50 ms using a step size of $5 \mathrm{~ms}$ (see also Fig. 7A). As the LFP traces were low-pass filtered at $25 \mathrm{~Hz}$ and the spiking responses were convolved with a density function, the exact size of the bins has only a minor influence on the correlation results. To compare stimulus-related correlations across individual sites, we first identified the time of the maximum correlation within a poststimulus onset window of $100-300 \mathrm{~ms}$ per site. Afterward, we extracted the average correlation within a window of $50 \mathrm{~ms}$ centered on the maximum of the correlation (see also Fig. $7 B)$. We performed this analysis separately for each color condition. Mean correlation coefficients did not differ significantly between color conditions (nonparametric Kruskal-Wallis test, V4: $X^{2}=0.14$ and $0.71, p>0.05$ for monkeys 1 and 2; PF: $X^{2}=3.16$ and $0.42, p>0.05$ for monkeys 1 and 2, respectively).

Selectivity analysis of spiking activity. To assess selectivity of spiking activity for different levels of coherence, we computed the d-prime estimate between all possible pairs of the different levels of coherence ( 0 vs 45,0 vs 55 , etc.) for each color condition individually. The d-prime value represents the mean of the trial differences in spiking activity between, for example, the $0 \%$ coherence and $100 \%$ coherence divided by the SD across trials in both conditions. Figure 9 shows maximal d-prime values obtained from comparison across all possible pairs of levels of coherence $(0-45,55-100$, etc.) across all color conditions.

\section{Results}

V4 and IPF are thought to play major roles in sensory and mnemonic processing of visual information within a hierarchical cortical network of visual object processing (Ungerleider and Pasternak, 2004). V4 lies at the intermediate stage of the visual hierarchy and has been implicated in processing color and shape information (Schein and Desimone, 1990; Zeki, 1980, 1983b; Desimone and Schein, 1987; Pasupathy and Connor, 2002; Gustavsen and Gallant, 2003; Rainer et al., 2004). IPFC is situated downstream from $\mathrm{V} 4$, and neurons in this region are highly modulated by the informational content and behavioral relevance of visual stimuli (Miller et al., 1996; Rao et al., 1997; Rainer et al., 1998; Miller, 
2000; Rainer and Miller, 2000, 2002; Zaksas and Pasternak, 2006). Based on the distinct visual properties of both regions obtained from single cell studies, we wondered whether visual information is similarly represented in the VEP component of the LFP recorded in both areas.

We simultaneously recorded spiking activity of single neurons and local field potentials while two rhesus monkeys were participating in a visual recognition task involving natural images. We chose natural images as our stimuli as they most closely represent stimulus conditions encountered in the natural visual environment (Felsen and Dan, 2005). We parametrically manipulated color and image structure of the images to test whether VEPs show similar color and shape modulations as single neurons do in V4. In addition, the degradation of our stimuli with visual noise allowed us to examine whether VEPs in prefrontal cortex modulate with the manipulation of task-relevant informational content in natural images similarly as has been described for prefrontal neurons.

\section{Stimulus conditions and behavior}

Figure 1 illustrates the different stimulus conditions for one example stimulus that was used in the current experiments. The image degradation procedure has been used in a range of studies to assess how information about visual stimuli affects neural processing in V4 and prefrontal cortex (Miller et al., 1996; Rainer and Miller, 2000; Rainer et al., 2004; Lee et al., 2005). In brief, all images were first normalized to have identical amplitude spectra in Fourier space resulting in matched spatial frequency content and contrast. We parametrically varied the amount of visual noise by combining the Fourier phase spectra of the natural images with a random phase spectrum, using the inverse Fourier transform at four coherence levels $(0 \%, 45 \%, 55 \%$, and $100 \%)$ to obtain the pure noise, intermediate noise, and full image conditions. Phase coherence is directly related to the level of degradation and we use the term "image degradation" and "stimulus coherence" interchangeably throughout this paper.

In the current study, we added a new element to the previously used degradation approach by also manipulating the color of our stimuli (Liebe et al., 2009). Figure $1 A$ shows the sequence of trial events during a trial of the delayed-matching-to sample (DMS) task. Figure $1 B$ shows an example image that was shown as a sample stimulus for the different stimulus conditions. The image was degraded with different amounts of visual noise (\% stimulus coherence) and shown in each of the color conditions A-C. Specifically, in color condition A ("achromatic condition"), we mixed achromatic noise with achromatic images. Consequently, information about the stimulus is solely based on shape defined by luminance cues. In condition B ("colored image condition"), we mixed achromatic noise with colored natural images. Here, we varied the amount of information based on image color as images are degraded with visual noise. At a given coherence level, information based on luminance-defined shape is similar for conditions A and B. Thus, a change in neural activity between these two conditions primarily reflects a contribution of image color. In a third condition C ("colored noise condition"), we mixed chromatic noise and achromatic natural images. Here, color is present in form of visual noise and does not provide task-relevant information. A comparison between conditions $\mathrm{B}$ and $\mathrm{C}$ allows us to assess whether color per se, i.e., color that does not carry task-relevant information, alters neural activity to the same extent as the task-relevant color in condition B. Figure $1 C$ shows the behavioral performance of the monkey subjects participating in this study during electrophysiological data acquisition.
The performance of the animals has been reported in detail in a previous report (Liebe et al., 2009). Similar to our previous findings we observed that recognition performance monotonically depended on stimulus coherence and that monkeys showed significant differences in recognition performance between color conditions A-C. Specifically, in both monkeys performance was improved for the colored image condition (B) and impaired for the colored noise condition $(\mathrm{C})$ relative to the achromatic condition (A) (psychometric thresholds $T$ for all color conditions estimated at $75 \%$ correct: monkey 1: $T_{\mathrm{A}}: 53.2,95 \%$ confidence intervals $\mathrm{ci}_{95}: 51.5$ and $55.1 ; \mathrm{T}_{\mathrm{B}}: 50.9, \mathrm{ci}_{95}: 49.5$ and $52.2 ; \mathrm{T}_{\mathrm{C}} 67.3$, $\mathrm{ci}_{95}: 60.8$ and 76.2, monkey 2: $T_{\mathrm{A}}: 50.4, \mathrm{ci}_{95}: 49.5$ and 51.47; $T_{\mathrm{B}}$ : $46.02, \mathrm{ci}_{95}: 45.7$ and 46.6 ; $T_{\mathrm{C}}: 53.4, \mathrm{ci}_{95}: 52.6$ and 54.6).

\section{Visually evoked potentials extracted from local field potentials are highly color sensitive in $\mathrm{V4}$, whereas in prefrontal cortex evoked responses are modulated by natural image structure}

We asked whether there were any systematic differences in visualevoked potential amplitudes recorded in area V4 and PF for the different stimulus conditions. Although traditionally the term stimulus tuning is used to refer to the selective response of a neuron along a single parametrically varied stimulus dimension, we use the term stimulus tuning throughout this paper to describe the sensitivity of VEP and SUA to changes in phase coherence and color of natural images.

Representative examples of single sites simultaneously recorded in each area are shown in Figure 2. (Additional examples are shown in supplemental Fig. 1, available at www.jneurosci.org as supplemental material). Each of the traces in Figure 2 corresponds to the trial-averaged visual-evoked potential for one of the stimulus conditions shown in Figure 1. In both monkeys and brain areas, VEPs were modulated with our stimulus manipulations but in a qualitatively different way. In V4, color modulated the amplitude of the evoked response. Specifically, amplitudes increased as coherence increased in condition B, and amplitudes decreased as coherence increased in condition C. This pattern is consistent with a systematic dependence of the VEP on the amount of color in the images, since for condition B color increases, and for condition $\mathrm{C}$ color decreases as the level of coherence changes from 0 to $100 \%$ (two-way repeated-measures ANOVA across trials with factors color condition, $F_{\mathrm{C}}>8.1, p<$ 0.001 , and factor level of coherence, $F_{\text {coh }}<2.3, p>0.05$, and both sites showing significant interaction effect with $F_{\text {int }}>4.8$, $p<0.001)$. Consistent with color being the primary determining factor, V4 VEP amplitudes showed no systematic modulation with coherence in the achromatic condition. For visually evoked potentials recorded in PF cortex, the pattern of results was qualitatively different. For the shown example site, VEP amplitudes increased with increasing coherence. This effect was similar in all color conditions A-C $\left(F_{\text {coh }}>15.7, p<0.001 ; F_{\text {col }}<0.8, p>\right.$ $\left.0.05 ; F_{\text {int }}<1.1, p>0.05\right)$. This suggests that color did not have a large impact on VEPs at this example site, but that image structure primarily determined PF neural responses. Together, these examples suggest that in V4 visual-evoked amplitudes of single LFP sites systematically increase with the amount of color in natural images, whereas visually evoked potentials of single sites in prefrontal cortex increase as a function of natural image structure, regardless of the color conditions.

To assess stimulus selectivity at the population level, we analyzed the LFP amplitude across V4 and PF sites within a $50 \mathrm{~ms}$ window surrounding the maximum negative peak in a poststimulus period window of $100-300 \mathrm{~ms}(N=32$ and 78 for V4 
A

Monkey 1

achromatic (a)

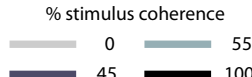

$45-100$
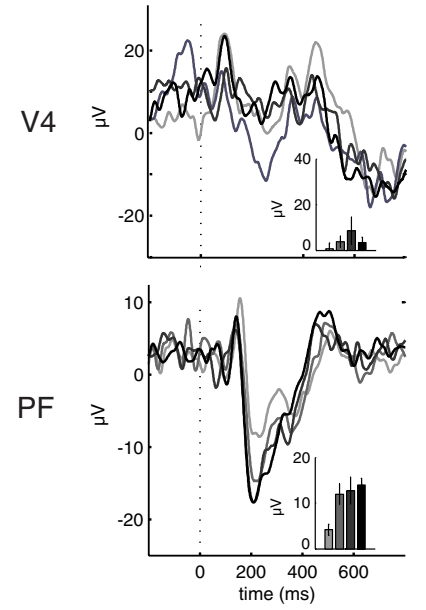

B

Monkey 2

V4
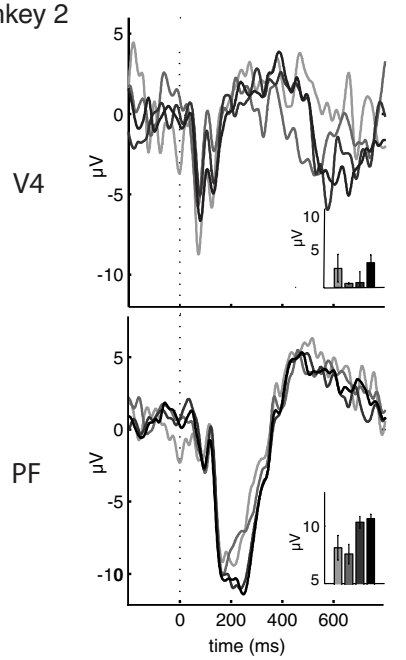

colored image (b)
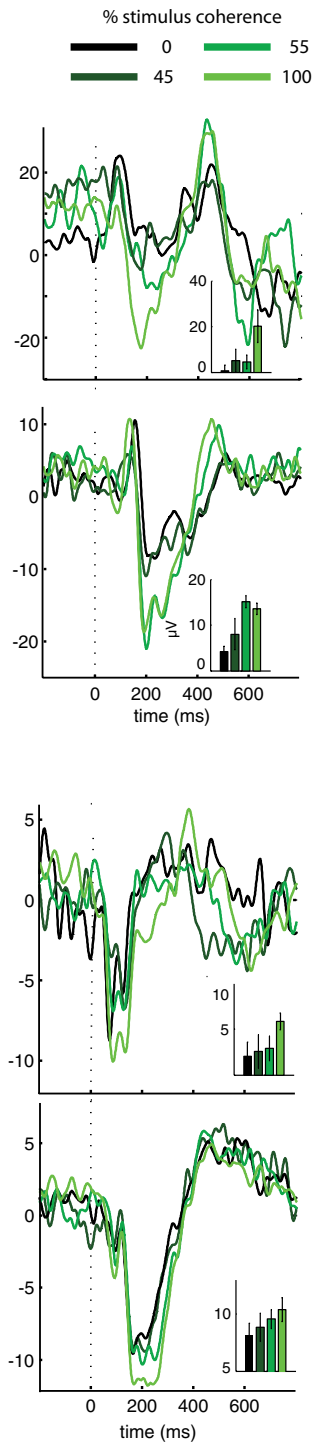

Figure 2. Representative examples of single LFP sites and their VEPs for the different stimulus conditions. $\boldsymbol{A}, \boldsymbol{B}$, In monkey $1(\boldsymbol{A})$ and monkey $2(\boldsymbol{B})$ for sites concurrently recorded in V4 and PF cortex. In each column of the graph, VEPs for different levels of coherence in the achromatic condition $(\boldsymbol{a})$, the colored image condition $(\boldsymbol{b})$, and the colored noise condition (c) are shown. Bar plots represent the average activity within a $50 \mathrm{~ms}$ window centered at the maximum peak amplitude $100-300 \mathrm{~ms}$ after stimulus onset. In V4, color leads to a systematic increase in the VEP, whereas luminance-based shape coherence does not. In PF cortex, VEP amplitude increases with increasing levels of coherence similarly for the different color conditions.

and $N=41$ and 66 for PF, for monkey 1 and 2, respectively). When averaging the peak activity across all sites in V4 and PF, we observed the same effect as for the individual sites. The results are summarized in Figure 3. For both monkeys, amplitudes in V4 varied systematically with color. This effect was significant in both animals, although it appeared slightly less pronounced in monkey 2 compared with monkey 1 across the population. (V4 $\left.F_{\mathrm{Col}}:>7.7, p<0.01 ; F_{\text {coh }}<1.5, p>0.05 ; F_{\text {int }}>3.7, p<0.01\right)$. Thus, the amplitude of VEPs was enhanced by color and did not show any systematic modulation with changes in phase coherence in the achromatic condition A. We confirmed this in a separate analysis on condition A for which we found no systematic modulation of VEPs with the level of coherence (one-way ANOVA $\left.F_{\text {coh }}<0.18, p>0.05\right)$. In contrast, in PF cortex image structure significantly influenced amplitudes across PF sites in a systematic fashion in both monkeys, and this effect was similar colored noise (c)
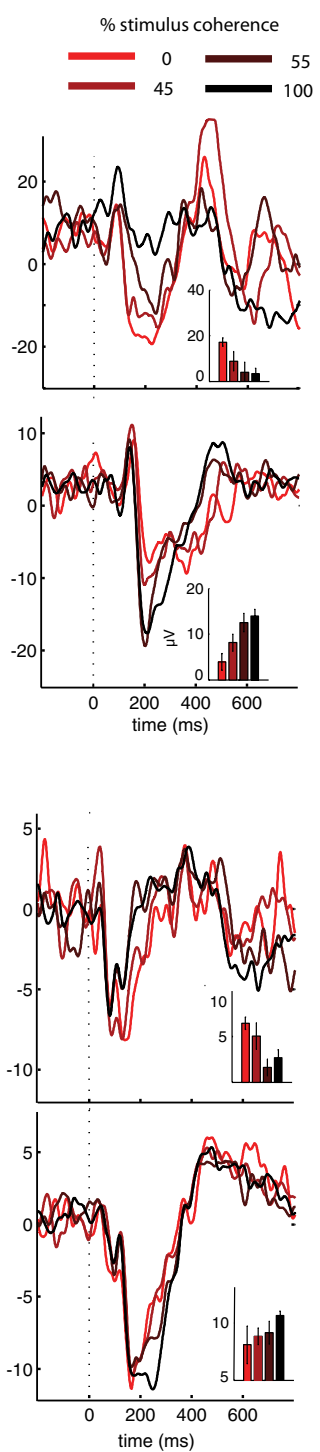

for all color conditions ( $\mathrm{PF} F_{\text {coh }}>32.5$, $p<0.001 ; F_{\text {col }}>2.62, p>0.05 ; F_{\text {int }}>2.5$, $p<0.01)$. In addition, we observed significantly higher VEP amplitudes for color condition $\mathrm{B}$ when compared with condition $\mathrm{A}$ and $\mathrm{C}$ at multiple coherence levels (see also asterisk symbols indicating significant differences in Fig. 3; multiplecomparison tests using Bonferroni correction, $t \gg 2.07, p<0.05)$. These increases in amplitude for condition $\mathrm{B}$ are consistent with the behavioral performance of the animals that showed significantly better recognition performance for condition $\mathrm{B}$ when compared with condition A and C (Liebe et al., 2009).

In summary, our population analyses revealed that color is the major determining factor of VEP amplitudes across the population of V4 sites, suggesting high color sensitivity at the mesoscopic level as measured by the LFP. In contrast, we found that VEP amplitudes in PF increase monotonically as a function of coherence for all color conditions similarly, suggesting that evoked LFP activity is rather tuned to image structure. In addition, behavioral advantages of monkeys in identifying degraded colored images (condition B) were reflected in PF VEPs.

\section{Single sites in V4 show consistent} sensitivity to color magnitude, whereas prefrontal sites are reliably modulated by visual noise

Next, we assessed whether the selectivity we found at the population level was characteristic of activity at single LFP sites. For example, it might be possible that different subpopulations of LFP sites are responsible for the respective increase and decrease in amplitude in conditions $B$ and C. On the other hand, if LFP sites are color sensitive regardless of whether the color is present as colored noise or image color, the same site should show increased and decreased amplitudes as coherence changes in conditions B and C, respectively. A distinction of these two possibilities is not feasible based on the averaged activity across sites. Thus, we fitted linear regressions to amplitude versus phase coherence functions for the different color conditions. Subsequently, we compared the slope parameter as an indicator for the type of selectivity across coherence levels. Positive slope estimates indicate that the amplitude is increasing with increasing phase coherence levels, whereas negative slope estimates indicate a decrease in amplitude as phase coherence levels increase. In Figure 4 we compared slope estimates obtained for conditions $\mathrm{B}$ and $\mathrm{C}$ within individual sites. In both monkeys, the majority of V4 sites exhibit positive slopes for condition B and negative slopes for condition $\mathrm{C}$, resulting in a significant clustering in the upper left quadrant (McNemar-test, $\chi^{2}>12.7, p<0.001$, binomial test using expected probability of 0.25 , monkey $1: 19$ and 32 , $p<0.01$, monkey 2: 29 and 78, $p<0.01$ ). In contrast, inspection 

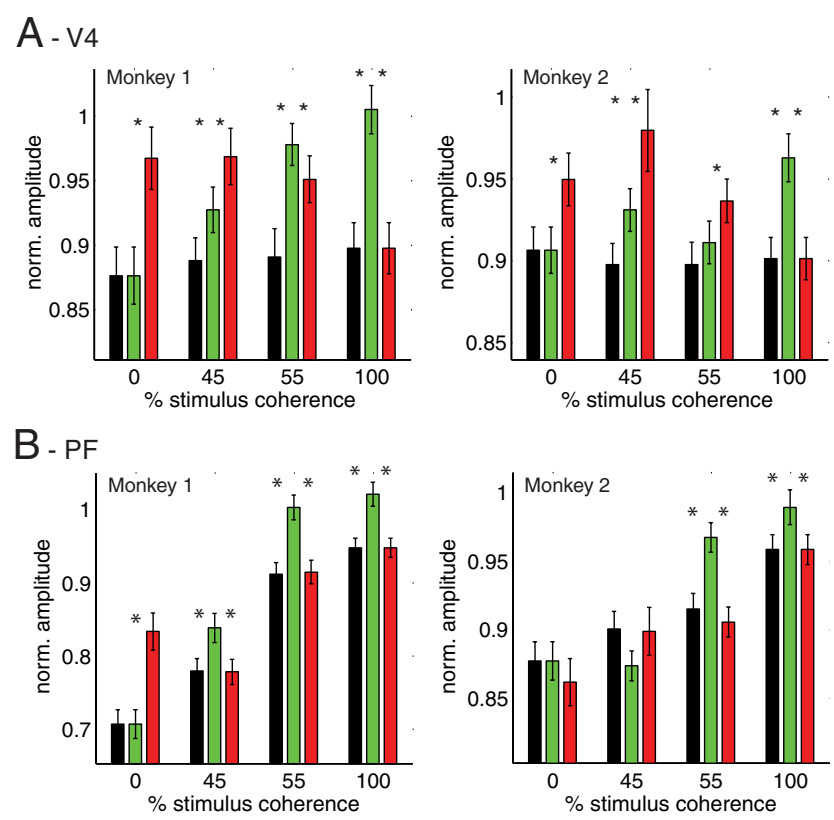

Figure 3. A, VEP amplitudes to different stimulus conditions averaged across recorded sites in V4. In both animals, VEP amplitudes are conversely tuned to color conditions B (green bars) versus C (red bars), indicating a strong influence on the amount of color magnitude. No systematic tuning the coherence levels can be found for the achromatic condition A (black bars). VEP amplitudes are normalized to the maximum amplitude per coherence condition after averaging across the color conditions separately for each recording site. Error bars denote $+/-1$ SEM. Asterisks denote significant differences between conditions. $\boldsymbol{B}$, VEP amplitudes to different stimulus conditions averaged across prefrontal sites. In both animals, VEP amplitudes are similarly tuned for the achromatic (black bars), colored image (green bars), and colored noise condition (red bars): VEP amplitudes are normalized to the maximum amplitude per coherence condition after averaging across color conditions separately for each recording site. Error bars denote $+/-1$ SEM. Asterisks denote significant differences between conditions.
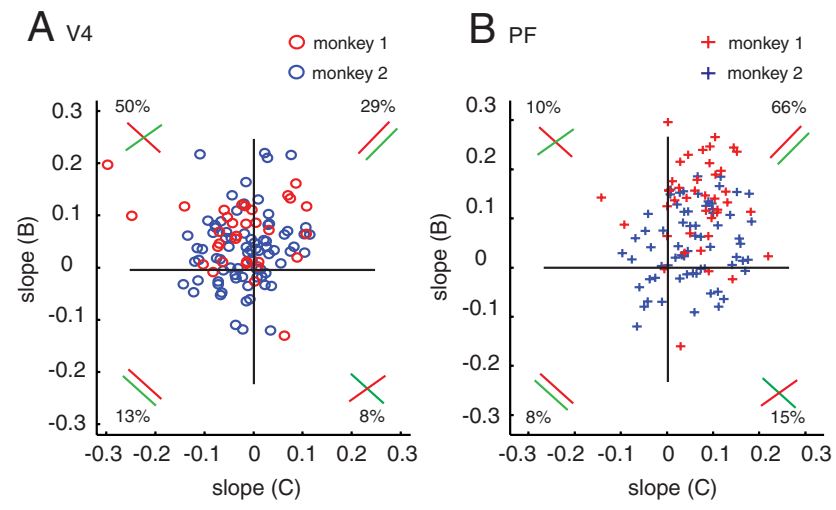

Figure 4. Distribution of slope parameters across LFP sites fitted to amplitude versus coherence functions comparing color conditions B (green lines) and C (red lines) in area V4 (circles, $A$ ) and PF cortex (crosses, $\boldsymbol{B}$ ). Blue and red symbols correspond to slope estimates from the two different animals. In both monkeys, the majority of sites in V4 is located in the upper left quadrant of the graph, which means that amplitudes increase with increasing coherence levels for condition B (positive slope estimates) and decrease with decreasing degradation levels for condition C (negative slope estimates) based on comparisons within individual sites. In contrast, the majority of sites in PF cluster in the upper right quadrant, which indicates that amplitudes similarly increase for conditions $B$ and $C$ as image degradation decreases when comparing within individual sites.

of Figure $4 B$ shows that in both monkeys the majority of sites in $\mathrm{PF}$ cortex are located in the upper right quadrant. This indicates positive slope estimates for both color conditions B and C, i.e., an increase in amplitude with increasing coherence levels $\left(\chi^{2}>\right.$
41.7, $p<0.0001$, binomial test, monkey 1: 35 of $41, p<0.001$, monkey 2: 39 of 66, $p<0.001$ ). These analyses confirm that responses observed at the example LFP site shown in Figure 2 are indeed representative of LFP activity in both V4 and PF cortex.

In conclusion, our results show that local field potential responses, as measured by magnitude of the evoked amplitude, show systematic modulations to changes in structure and color properties of natural images. In V4, the amplitudes highly correlate with the presence of color, showing no systematic selectivity for variations in luminance-based shape properties and no systematic effect with increases of shape coherence per se. In contrast, in PF cortex amplitudes are exclusively tuned to image structure, resulting in a similar enhancement in amplitudes for all three different color conditions as coherence is increased from $0 \%$ (pure noise stimuli) to $100 \%$ coherence (full images). Image structure always provides task-relevant information across the three color conditions, whereas color does not: it is absent in the achromatic condition, provides task-relevant information aiding image segmentation in the colored image condition, and causes task-irrelevant signals in the colored noise condition. Our results thus imply that VEP responses in PF cortex are tuned primarily to task-relevant informational content of images.

\section{Stimulus sensitivity is similar between averaged VEP and spiking activity in PF but not in V4}

The LFP is a slow-varying electric potential that reflects subthreshold dendritic activity around the recording electrode tip and is thus influenced by recurrent processing of the local spiking population as well as projecting inputs from other areas (Katzner et al., 2009; Mitzdorf, 1985; Mitzdorf, 1994). Thus, in contrast to suprathreshold spiking that mainly represents the output signal of a given area, LFPs rather measure subthreshold input into the region. Investigation of their respective stimulus sensitivities can reveal insights into cortical computation: if VEPs and single unit responses are similarly tuned, VEPs likely reflect subthreshold activity of the local spiking population within the recording area. If VEPs show qualitatively different selectivity than spiking activity, VEPs possibly reflect the properties of the input into the region arising from neurons located in projecting areas. In the following analyses we examine how similar stimulus-selectivity of VEP responses is compared with sensory characteristics obtained from simultaneously recorded spiking activity of single neurons. Given the robust and characteristic selectivity of VEP amplitudes to our stimulus conditions in area V4 and PF, we asked whether (1) we would find the similar color sensitivity we obtained for VEP amplitudes also for spiking activity in V4, and (2) whether spiking activity in PF behaves in a similar fashion compared with VEP amplitudes (i.e., showing an increase in activity as coherence levels increase).

Examples for image structure and color sensitivity of simultaneously recorded LFP-SUA pairs in V4 are shown in Figure 5. Additional examples are shown in supplemental Figure 2, available at www.jneurosci.org as supplemental material. Figure 5, $A$ and $B$, shows the average VEPs and PSTH during the sample period across color conditions $B$ and $C$ for a representative recording site in V4. To assess the dependence of neural activity on color, we sorted the trials of conditions $\mathrm{B}$ and $\mathrm{C}$ according to the magnitude of color contained in the images, not according to the level of coherence, i.e., the $100 \%$ coherence trials of condition B were sorted in the same group as the $0 \%$ coherence trials of condition $\mathrm{C}$ and so forth. Thus, color sensitivity will result in enhanced responses as color magnitude changes from 0 to $100 \%$. Figure $5 A$ shows a clear dependence of VEP amplitudes on color 


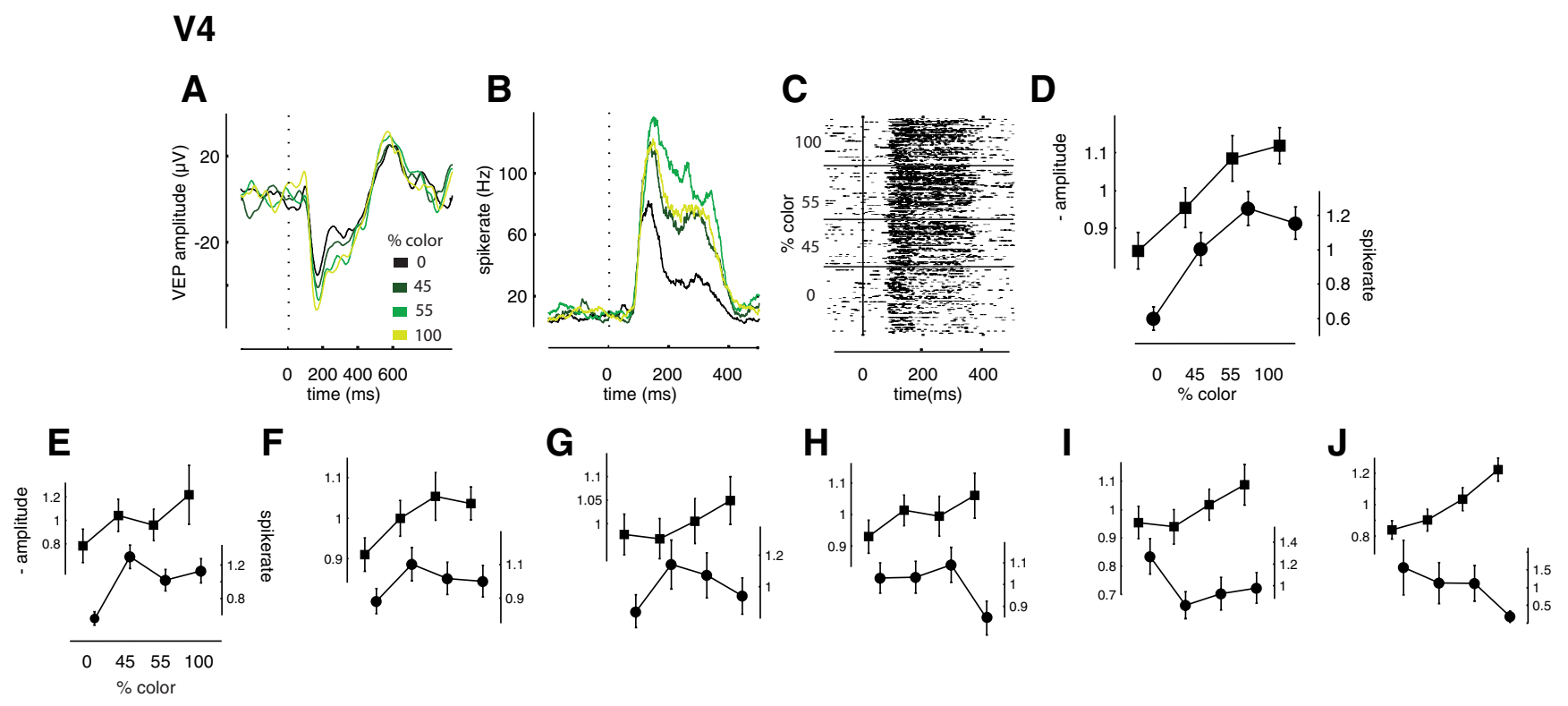

Figure 5. A-D, Representative example for color tuning of simultaneously recorded VEP and SUA in V4. A, Time-averaged VEP for different levels of color collapsed across the two color conditions. $\boldsymbol{B}$, Peristimulus time histogram of single unit recorded at LFP site shown in $\boldsymbol{A}$ for different color levels. $\boldsymbol{C}$, Raster plot of spiking activity for single unit shown in $\boldsymbol{B}$ for different color levels. $\boldsymbol{D}$, Averaged VEP amplitudes and spiking activity at different levels of coherence for LFP site and single unit shown in $\boldsymbol{A}$ and $\boldsymbol{B}$. Activity is normalized to maximum across coherence levels. Error bars denote $+/-$ 1 SEM across trials (rank correlation coefficient $c_{\text {rank }}$ for this example pair is 0.045 ). $\boldsymbol{E}-\boldsymbol{J}$, Additional examples for averaged VEP and single unit activity at different levels of color for monkey 1 ( $\boldsymbol{E}-\boldsymbol{G}$, $\left.c_{\text {rank }}:-0.02,0.021,-0.05\right)$ and monkey $2\left(H-J, c_{\text {rank }}: 0.002,-0.026,-0.06\right)$.

for the LFP recorded at this electrode site. In contrast, Figure $5 B$ shows that SUA recorded at that site is highest for the intermediate condition. Figure $5 C$ shows the activity of the same unit in a raster plot, which illustrates the variability of spiking activity per color level across all stimuli presented. Figure 5D summarizes the tuning of this electrode site and plots the normalized average VEP amplitude and single unit activity as a function of percentage color. Figure $5 E-J$ show similar plots from additional example sites from monkey $1(E-G)$ and monkey $2(H-J)$. When we examined the VEP and spike selectivity for single recording sites in V4, we did not find a clear correspondence between both types of signals. This was also true at the population level of spiking activity (supplemental Fig. 4, available at www.jneurosci.org as supplemental material): the color sensitivity that is evident in the average across V4 VEPs was not evident in average V4 spiking activity. Single unit responses did not generally increase as the amount of color increased, but rather displayed a variety of complex response functions, including inverted U-shaped tuning, which is similar to previous findings (Rainer et al., 2004).

In contrast, inspection of Figure 6 reveals that in PF, VEP amplitudes similarly increase as a function of coherence as SUA recorded at the same site. Similar to Figure 5, Figure 6, $A$ and $B$, shows the average VEPs and PSTH during the stimulus period across color conditions $\mathrm{B}$ and $\mathrm{C}$ for a representative recording site in $\mathrm{PPF}$. Here, trials were grouped according to coherence levels (i.e., the $0 \%$ coherence trials of condition $\mathrm{B}$ were sorted in the same group as the $0 \%$ coherence trials of condition $\mathrm{C}$, and so forth). Figure $6 C$ shows the activity of the same unit in a raster plot and illustrates the spiking responses as a function of coherence across all stimuli presented. For this example, VEPs and SUA increase similarly as a function of coherence. We observed similar response patterns in other LFP-SUA pairs, whose normalized activity is shown in Figure $6 E-G$ for monkey 1 and Figure $6 \mathrm{H}-\mathrm{J}$ for monkey 2. Additional examples are shown in supplemental Figure 3, available at www.jneurosci.org as supplemental material. These results were consistent when we compared the average spiking population response to the averaged VEP response: for both types of signals, neural activity increased as images became less degraded (see supplemental Fig. 4, available at www.jneurosci.org as supplemental material), suggesting similar stimulus sensitivity for single neuron response and stimulusevoked components of the LFP in IPF.

\section{Prefrontal units and VEPs show significant signal correlations during visual stimulation, which is not the case for V4 units}

To further quantify the relationship between VEP and spike sensitivity in V4 and IPF, we computed Spearman's rank correlation coefficients $\left(c_{\text {rank }}\right)$ between VEP amplitudes and spiking activity across the different coherence and color conditions for each pair of simultaneously recorded SUA and LFP responses. Specifically, we computed the rank correlation within a window ranging from $200 \mathrm{~ms}$ before to $800 \mathrm{~ms}$ after stimulus onset across the population of SUA-LFP pairs ( $N=58$ for V4 and 51 for PF). We should point out explicitly here that we are not computing the instantaneous similarity in VEP and spiking activity (so-called noise correlation). Instead, we shuffled the trials per condition and thus compute only the "signal" correlation, i.e., the similarity in spiking and VEP activity solely due to variations in the stimulus parameters.

The result is summarized in Figure 7A. For prefrontal LFPSUA pairs, we found a significant increase in correlation between SUA and VEP responses after stimulus onset compared with baseline, whereas for V4 LFP-SUA pairs this was not the case. We also compared the correlation across the population of LFP-SUA pairs between the baseline and the stimulus period for each monkey separately. Figure $7 B$ shows the distribution of correlation differences between stimulus period and baseline from LFP-SUA pairs in V4 (monkey 1 and 2, left/right panel, respectively). In both animals, the correlation coefficients did not significantly 

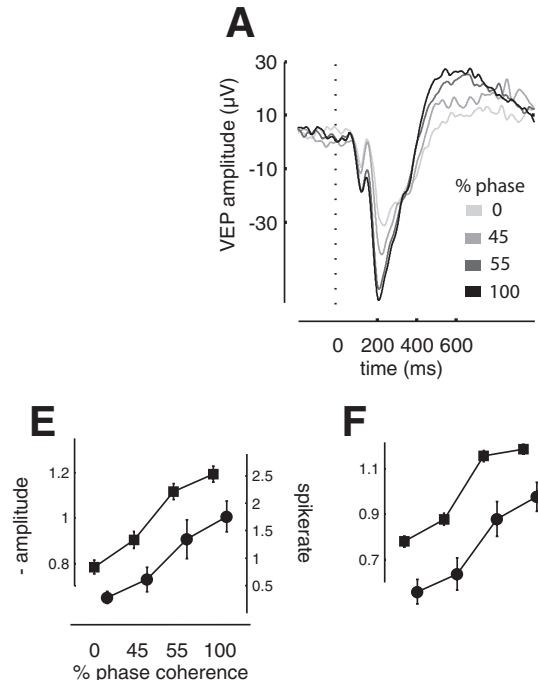

F

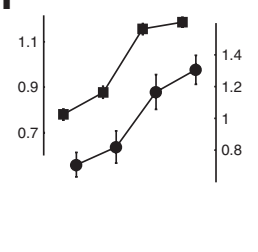

B

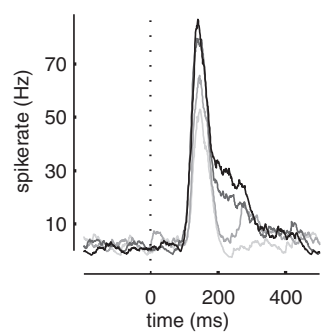

G

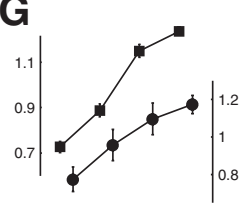

C
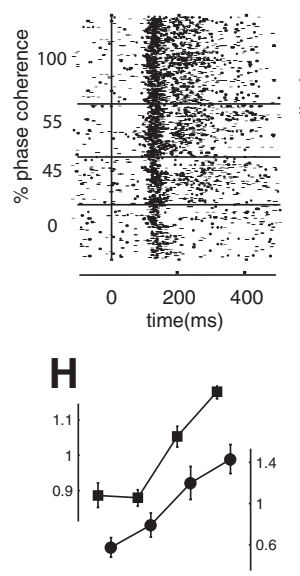

D

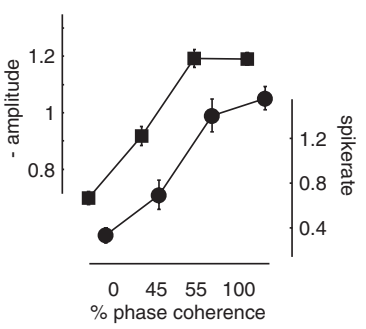

I

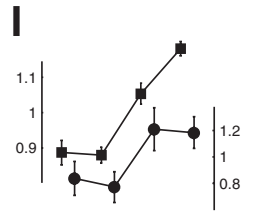

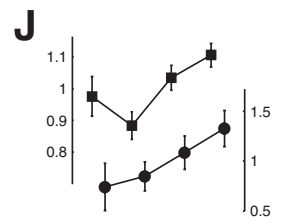

Figure 6. $\boldsymbol{A}-\boldsymbol{D}$, Representative example in PF for phase coherence tuning of simultaneously recorded VEP and SUA. $\boldsymbol{A}$, Time-averaged VEP for different levels of phase coherence collapsed across all color conditions. $\boldsymbol{B}$, Peristimulus time histogram of single unit recorded at LFP site shown in $\boldsymbol{A}$ for different levels of phase coherence. $\boldsymbol{C}$, Raster plot of spiking activity for single unit shown in $\boldsymbol{B}$ for different levels of phase coherence. $\boldsymbol{D}$, Averaged VEP amplitudes and spiking activity at different levels of coherence for LFP site and single unit shown in $\boldsymbol{A}$ and $\boldsymbol{B}$. Activity is normalized to maximum across coherence levels for the mean across color conditions. Error bars denote $+/-1$ SEM across trials. (Rank correlation coefficient $c_{\text {rank }}$ for this example pair is 0.21 .) $\boldsymbol{E}-\boldsymbol{J}$, Additional examples for averaged VEP and single unit activity at different levels of image degradation for monkey $1\left(\boldsymbol{E}-\boldsymbol{G}\right.$, rank correlation coefficient $\boldsymbol{C}_{\text {rank }}: 0.12,0.11$, and 0.09$)$ and monkey 2 ( $\boldsymbol{H}-\boldsymbol{J}$, $c_{\text {rank }}: 0.12,0.06$, and 0.05$)$.

differ between baseline and stimulus period (paired $t$ test, $t>1.6$, $p>0.05$ ). Figure $7 C$ shows the same results obtained from LFPSUA pairs in PF. Here, the correlation coefficients significantly differed between baseline and stimulus period for both animals $(t>2.3, p<0.05)$.

We also obtained similar results when we merged spike trains from all units recorded at one electrode to create "multiunit" activity (MUA). Since we tried to yield well isolated single unit activity in each of our recordings, we were only able to merge the activity of two single units on average at 12/11 of our recording sites, in IPF and V4, respectively. Although this procedure is somewhat different from analyzing MUA by high-pass filtering raw voltage traces, we could still compute stimulus correlation between VEPs and spike responses from this "surrogate" multiunit activity. The results are shown in supplemental Figure 5, available at www.jneurosci.org as supplemental material, which plots histograms of stimulus correlation coefficients resulting from single units and merged unit activity. Similar to single unit activity, stimulus correlation was significantly enhanced in PF but not V4 (paired $t$ tests: $t>2.3, p<0.05$ for PF and $t<1.9, p>$ 0.05 for V4). In addition, stimulus correlation between VEP and spiking activity was significantly higher for the constructed multiunits than for single units in lPF but not V4 (ranksum test: lPF, $Z=2.2, p<0.05 ; \mathrm{V} 4, Z=0.3, p>0.05)$.

Our previous analyses focused on the question of whether we would find the same color sensitivity we obtained for VEP amplitudes also for spiking activity in V4, and whether spiking activity in PF similarly increases as images become less degraded. To investigate this, we sorted the trials according to what stimulus parameters VEPs were most sensitive to. Another interesting question is whether the stronger similarity in tuning between SUA and VEPs in PF is due to a more general correspondence in stimulus-dependent neural activity within this region. Thus, we asked whether we also find stronger correspondence between
SUA and VEP responses in PF if we do not arrange trials according to their prominent VEP selectivity. To this end, we computed rank correlations between single trial activity of spike responses and VEPs across all 12 stimulus conditions. This way, the correlation describes the general similarity in stimulus-dependent variations of SUA and VEPs disregarding their qualitative sensitivity to particular stimulus features. The results are summarized in supplemental Figure 7, available at www.jneurosci.org as supplemental material. Again, we found significantly enhanced stimulus correlation in PF, but not V4 (PF, $t>2.79, p<0.01$; V4, $t<$ $1.89, p>0.05)$. These findings confirm that stimulus-dependent visually evoked SUA and LFP activity is in general more similar in $\mathrm{PF}$ than inV4, at least for the stimulus parameters tested.

\section{In prefrontal neurons, signal correlation is largely absent during the delay}

One striking characteristic of prefrontal spiking activity is that neurons often exhibit delay activity within the context of visual recognition tasks that can be selective for different stimulus conditions (Fuster and Alexander, 1971; Miller et al., 1996). Although spiking activity during the delay is less dependent on sensory events, and LFPs during the delay are generally not characterized by externally driven time-locked components as can be observed during the sensory period, we still wondered whether stimulus-evoked activity is similar between LFPs and spiking during the delay, especially given the robust similarity in stimulus selectivity we found within the visual response window. When we computed signal correlations across the entire trial period for units showing or not showing enhanced activity during the delay period ( $N=24$ and 27, respectively, based on paired $t$ tests, $p<$ 0.05 ), we observed that both groups of units showed significantly enhanced signal correlation after sample onset $(t>2.2, p<0.05)$, but not during the delay (defined as the last $1000 \mathrm{~ms}$ preceding test stimulus onset, $p>0.05$ ) (Fig. 8). However, several SUA-LFP 
A

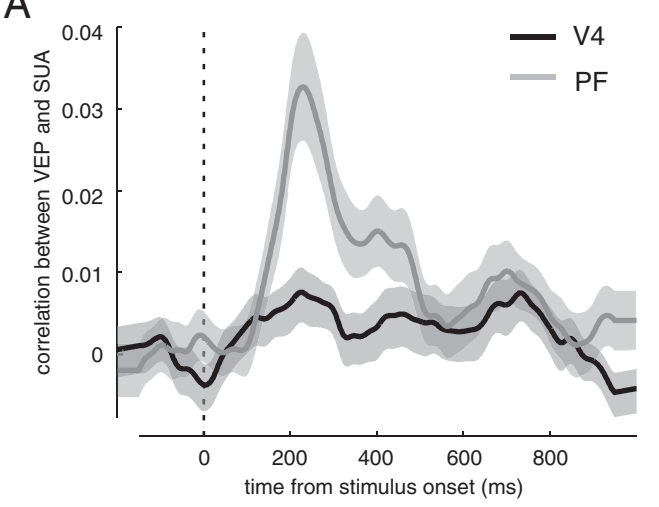

B-v4 monkey 1

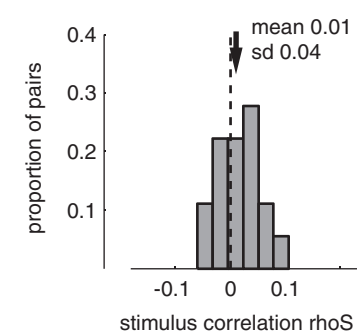

monkey 2 mean 0.005
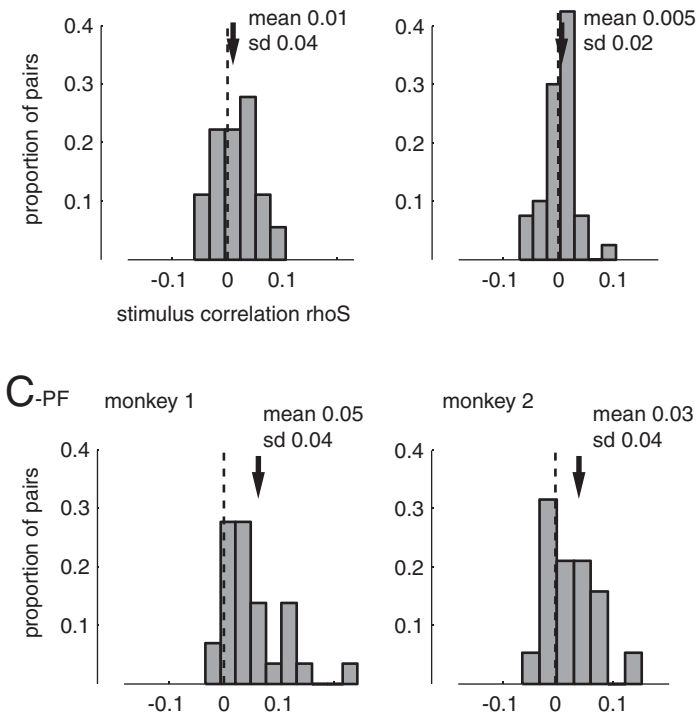

Figure 7. A, Mean correlation coefficient computed across time for all pairs with simultaneously recorded VEP and spiking activity in V4 (black) and PF (gray). Whereas the stimulus correlation between VEP and spiking activity for prefrontal cortex rose during stimulus presentation, it does not in V4. Error bars (shaded area) denote $+/-1$ SEM. B, Distribution of stimulus correlation between VEP amplitudes and spiking activity for simultaneously recorded pairs in area V4 (difference between stimulus period and baseline). For both monkeys, the correlation between VEP amplitudes and spiking activity across degradation levels was not significantly larger during the stimulus period than during the baseline. C, Distribution of stimulus correlation between VEP amplitudes and spiking activity for simultaneously recorded pairs in area PF. For both monkeys, the correlation between VEP amplitudes and spiking activity across coherence levels was significantly larger during the stimulus period compared with the baseline.

pairs (three in monkey 1 and four in monkey 2) exhibited a significant correlation during the delay period. Spiking activity recorded at these sites systematically varied as a function of stimulus coherence. The LFP was characterized by general negative amplitude shifts as a function of stimulus level (analog to DC shifts), which was qualitatively similar to evoked LFP activity during the visual presentation of the stimulus (two example pairs are shown in supplemental Fig. 8, available at www.jneurosci.org as supplemental material). Together, these findings show that stimulus-correlations between the evoked components of the two signals are weaker during the delay than stimulus presentation and thus are task dependent. However, this effect can likely be explained by the fact that externally driven time-locked modulations in LFP amplitudes are largely absent during the delay period. Here, analyses based on oscillatory components of ongo-
A

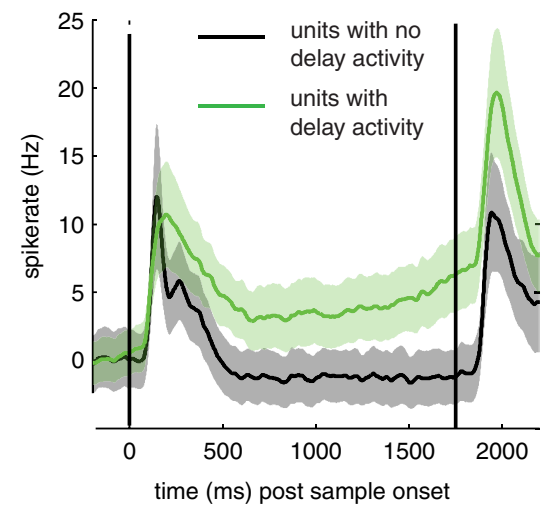

B

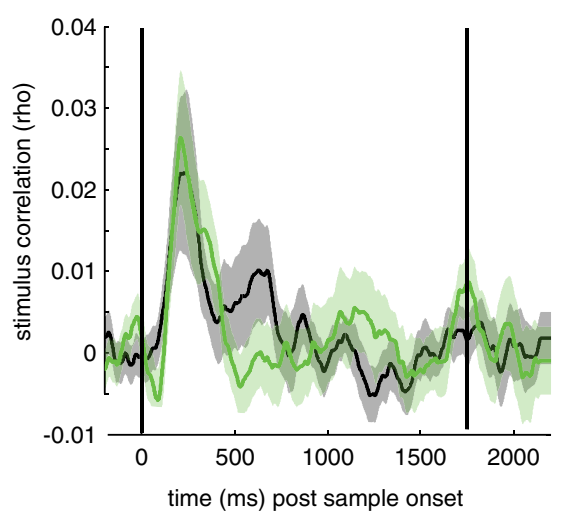

Figure 8. PSTH $(\boldsymbol{A})$ and stimulus correlation $(\boldsymbol{B})$ for prefrontal units showing significant delay activity (green, $N=24$ ) or not (black, $N=27$ ). Both groups of units show significantly elevated stimulus correlation during sample stimulus period ( $t=2.8$ and 2.2, $p<0.05$ for delay and non-delay units, respectively; the onset of the sample is indicated by a black line at 0 $\mathrm{ms})$. In addition, neither group shows enhanced stimulus correlation during the delay (delay period last 1000 ms before test stimulus onset, $t=0.5$ and $-0.3, p>0.05$ for delay and non-delay units, respectively).

ing LFP fluctuations might reveal further insights into how stimulus conditions influence the relationship between spiking activity and LFPs during the delay.

\section{The dissociation in stimulus tuning between SUA and VEPs in $\mathrm{V} 4$ is not due to a lack in stimulus selectivity of single neurons}

In the previous analyses we showed that stimulus sensitivity seemed to be more similar between SUA and VEPs in PF than in V4. However, a lack of correlation between LFP and stimulus tuning could also arise from an overall lack of stimulus tuning in the spike responses. If SUA activity is not modulated by our stimulus conditions but only VEPs, this would also result in a lack of correlation. To test this possibility, we computed the selectivity of single units between different levels of coherence at different color conditions and plotted the selectivity against the correlation coefficients between LFP and spike responses. Thus, we were able to assess, first, whether single unit activity is tuned at all, and, second, whether neurons that show a greater correlation to VEP tuning are also themselves more selective. If this is true, there will be a clear relationship between selectivity indices and correlation coefficients. However, this will not be the case if single units are selective but show no correspondence to VEP selectivity. In this case the selectivity indices will be low (and the symbols will be located at the bottom of the graph). Figure 9 shows that the 


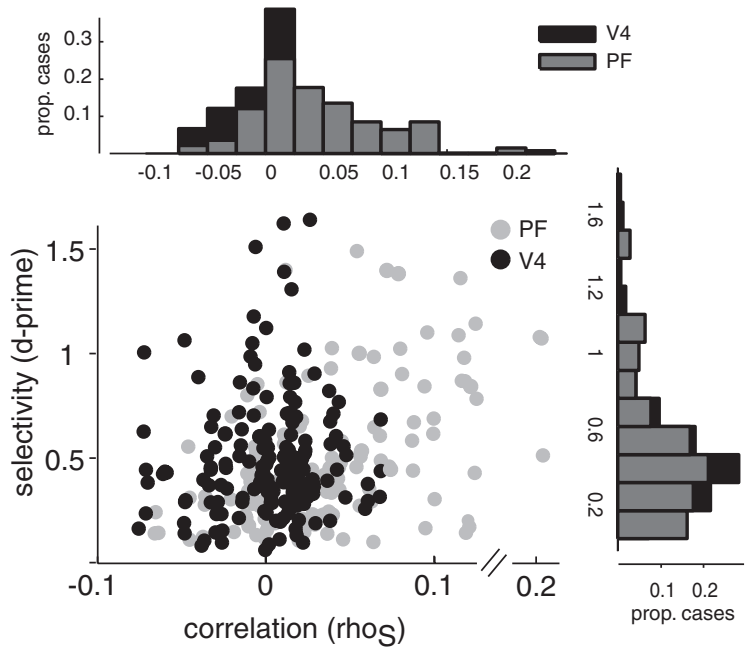

Figure 9. VEP-SUA correlation versus SUA-selectivity between different stimulus coherence levels in V4 (black) and PF (gray) across both animals. Histograms (top and right) show respective distributions of selectivity indices (d-prime values) and rank correlation coefficients for V4 (black) and PF (gray) across both monkeys. Whereas the magnitude of selectivity is similar in PF and V4 (two-sample $t$ test: $t=-0.36, p>0.05$ ), only in PF did we find a correlation between VEP responses and SUA with respect to stimulus tuning (ranksum-test comparing rank correlation: $Z=7.4, p<0.001$ )

magnitude of selectivity between stimulus coherence levels is similar in PF and V4, which results in higher correlation coefficients corresponding to higher selectivity values in prefrontal LFP-SUA pairs. This indicates similar qualitative tuning for VEP and single unit activity (Spearman rank correlation $c_{\text {rank }}>0.4$, $p<0.001)$. In contrast, most of the V4 pairs are located along the vertical axis of the graph, indicating that selectivity is not similar between VEP amplitudes and single unit activity $\left(c_{\text {rank }}<0.15\right.$, $p>0.05)$. Thus, in V4 the lack of stimulus correlation between LFP and SUA is not due to the fact that V4 neurons are not selective per se, i.e., show no response modulation to our stimulus manipulations but, unlike VEPs, are not generally modulated by enhancements in color.

\section{Stimulus selectivity in PF is independent of reward}

Finally, one factor that needs to be taken into account when interpreting our results of prefrontal activity is the fact that the degradation level of the sample stimuli is associated with the reward the animals receive. An increase in amplitude of the VEP as well as spiking activity in $\mathrm{PF}$ in response to the sample stimulus could thus also reflect an increase in reward expectancy. Indeed, neurons in lateral prefrontal cortex modulate their responses with the associated reward of stimuli, in addition to their identity and location (Leon and Shadlen, 1999; Wallis and Miller, 2003; Wallis, 2007). However, many of these neurons do not encode expected reward in a similar fashion. In addition, the responses of many neurons are not modulated in a parametrical way, i.e., neurons do not show simply increased or decreased activity as a function of reward magnitude. This is in contrast to our findings, in which the vast majority of neurons and LFP sites systematically increase their activity as stimulus coherence increases. To examine whether reward expectancy could still be responsible for the increase in response magnitude we find in $\mathrm{PPF}$, we compared neural responses to test and sample stimuli presented at $100 \%$ stimulus coherence. We found that SUA and VEP responses to test stimuli are highly correlated with responses to $100 \%$ coherence sample stimuli (rank correlations $\gg 0.88, p<0.001$ for both animals and types of signals), and identical stimuli evoked similar activity during the sample period and the test stimulus period (Fig. 10). As test stimuli are not associated with reward, since they occur on both match and nonmatch trials, these findings indicate that task-relevant visual information rather than the associated reward primarily determines prefrontal responses.

\section{Discussion}

We investigated sensory characteristics of spiking activity and visual-evoked components of the LFP in two cortical regions that are critical for visual object processing, the intermediate visual area V4 and the lateral prefrontal cortex. By concurrently measuring spiking activity and VEPs in both areas in response to identical natural image manipulations, we were able to assess whether and how tuning properties of VEPs and single units are related.

\section{In V4 we observed that stimulus selectivity differed between} VEPs and spiking activity

VEPs were sensitive to the magnitude of color in natural images revealing a strong functional specialization for color processing at the level of dendritic activity in V4. While our study is the first systematic investigation of color LFP signals in monkeys, our results are similar to studies demonstrating color sensitivity of VEPs in human V4 (Allison et al., 1993; Buchner et al., 1994; Murphey et al., 2008), and also support many studies using functional imaging both in humans and nonhuman primates that highlight the significance of V4 in color processing (Lueck et al., 1989; Bartels and Zeki, 2000; Conway and Tsao, 2006; Conway et al., 2007; Tanigawa et al., 2010).

In contrast to VEPs, the averaged spiking population response did not systematically modulate with color magnitude. Accordingly, we did not find significant stimulus correlations between VEPs and spiking activity. The lack of color sensitivity at the population level in V4 seems to be at odds with previous studies recording single unit activity that illustrate the importance of V4 in color processing (Zeki, 1980, 1983a,b; Wild et al., 1985; Walsh et al., 1993; Kusunoki et al., 2006; Stoughton and Conway, 2008; Conway and Tsao, 2009). Instead, our findings might rather be consistent with studies questioning a specific role of V4 neurons in color processing (Schein et al., 1982; Tootell et al., 2004). However, although there was no general increase in spiking activity across the neuronal population as a function of color magnitude, V4 neurons still exhibited multifaceted color selectivity at the single cell level, which highlights their importance in color processing. Our findings also fit well with the idea that V4 neurons do not exclusively encode a single stimulus feature, for example one particular color, but likely represent multiple features or the combination of features (Schein and Desimone, 1990; Kobatake and Tanaka, 1994), a characteristic that would be especially prominent in natural scenes, in which multiple features are simultaneously represented within one image.

In $\mathrm{PPF}, \mathrm{VEPs}$ as well as spiking responses varied systematically with image degradation: neural activity as measured by VEPs and SUA was similarly enhanced for stimulus conditions with enhanced image structure, which correlated with higher informational content and enhanced behavioral performance of the animals. Accordingly, stimulus correlations between both types of signals were significantly increased during stimulus presentation. Our results on VEP selectivity are consistent with previous studies recording spiking activity in $\mathrm{PPF}$, reporting that the prefrontal cortex is involved in encoding stimuli in terms of behavioral relevance rather than their physical identity (Rainer et al., 

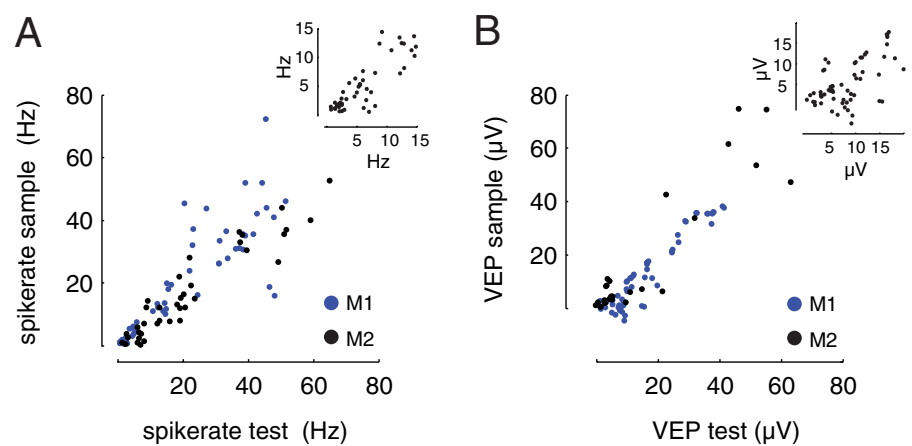

Figure 10. SUA and VEP responses to test stimuli are highly correlated with responses to $100 \%$ coherence sample stimuli. $A$ and $\boldsymbol{B}$ show visual responses, i.e., averaged SUA/VEPs within $100-250$ ms poststimulus onset for test ( $x$-axis) and $100 \%$ sample ( $y$-axis) stimuli (both achromatic and colored images). The small scatter plots in the upper left corner of the graphs zoom into the lower data range. Differently colored symbols represent different animals. For both types of signals and animals, we found a highly significant correlation between the activity for the sample and the test. C and $\boldsymbol{D}$ show mean SUA/VEP responses during sample and test presentation for colored and achromatic images; no significant differences were observed.

1998; Asaad et al., 2000; Freedman et al., 2001). Along these lines, our findings support the notion that prefrontal activity is less determined by an intrinsic specialization for processing particular stimulus attributes but is rather characterized by a flexible and learning-dependent adaptation to behaviorally important features (Yajeya et al., 1988; Desimone and Duncan, 1995; Miller, 2000; Duncan, 2001; Miller and Cohen, 2001). In our stimuli, these features could have been regions of the image by which the animals learned to recognize them, even in the presence of visual noise.

Another factor that needs to be taken into account when interpreting our results is the role of attention. It has been shown for several cortical areas (including V4) that directing subjects' attention to a particular stimulus feature modulates neural activity, mainly by increasing activity for attended stimulus features (Desimone and Duncan, 1995; Treue and Maunsell, 1996; Maunsell and Cook, 2002). For example, the animals' attention to particular stimulus features could be higher for non-degraded images than degraded ones, simply because they can be "seen" more easily and thus attract more attention. This effect could have contributed to the enhanced neural activity in IPF as images became less degraded. In contrast, the hypothesis that increased attention for non-degraded images leads to enhanced neural activity cannot be easily reconciled with our results in V4. In V4, SUA and VEP responses did not systematically vary with the level of image degradation. For example, we found increased evoked potential amplitudes for colored images while in the colored noise condition we observed decreased amplitudes as stimuli became less degraded. Thus, attention is unlikely to have systematically affected neural activity in V4 in our task design. Although it is still possible that the attentional state of the animal could have affected neural activity in a nonspecific way, the modulation of neural activity by our stimulus manipulations seems to be the more parsimonious explanation for our findings in V4.

Together, we observed distinct sensory characteristics of visual-evoked components of the LFP in both V4 and lateral prefrontal cortex. More interestingly, we found that sensory properties of VEPs and spiking activity differed in area V4, whereas they were similar in IPF.

The similarity between LFP and spike-selectivity in IPF is compatible with several studies reporting close resemblance in sensory tuning between the two types of signals, for example testing orientation tuning preference in macaque primary visual

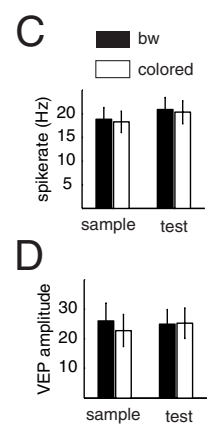

cortex (Katzner et al., 2009; Xing et al., 2009). Along these lines, several studies showed that the correlation between LFP and SUA selectivity also depends on whether or not similarly tuned neurons are homogeneously arranged in cortical space: if neurons surrounding the recording site are similarly tuned, the LFP selectivity will correspond to the that of single neurons (Kulikowski et al., 2002; Schummers et al., 2002; Nauhaus et al., 2008). Based on these findings, it has been suggested that LFPs reflects the averaged local spiking population response. This idea could potentially explain our findings in IPF. If single neurons are similarly tuned to informative features of our images, sensory characteristics between single neuron activity and LFPs would be related.

In contrast, the idea that the evoked components of the LFP represent the local spiking population is not compatible with our findings in V4. Here, on average, spiking activity did not increase with color magnitude, but visually evoked potentials did.

In dorsal V4, several studies have shown that neurons with different color preferences are distributed heterogeneously in cortical space (Zeki, 1983c; Tanaka et al., 1986; Stepniewska et al., 2005; Conway and Tsao, 2006). Based on these studies, one can assume that differently tuned neurons contribute to the visualevoked LFP responses that we measured. If the LFP merely reflects average local spiking activity one would expect both signals, mean spiking and mean VEPs, to not show a systematic modulation in response to variations in color magnitude.

A more recent study, however, presented evidence for a finescale spatial organization of color preference in adjacent neurons that were located in regions containing a high number of colorselective cells in posterior inferior temporal cortex, including area V4 (Stoughton and Conway, 2008). However, the similarity in color preference was most evident for cortical distances lower than $50 \mu \mathrm{m}$. Given that the LFP likely integrates neural activity across a radius of $\sim 250 \mu \mathrm{m}$ (Katzner et al., 2009), the LFP would represent the activity of neurons with heterogeneous color preferences and is thus spatially too coarse to be able to reflect a fine-scale organization in color preferences.

Alternatively, the fine-scale neuronal homogeneity of color preferences could be restricted to regions with a high density of color "globs," for example the posterior bank of the superior temporal sulcus, as opposed to the dorsal part of V4 located on the surface of the prelunate sulcus; the same region that we targeted with our recordings and that has been shown to have fewer regions with clear color preference (Conway and Tsao, 2006; Conway et al., 2007). Thus our findings might indicate that color processing in V4 is distinct within different subregions, an idea that has been put forth previously (Zeki, 1983c).

On the other hand, the dissociation we found between spike and VEP selectivity in V4 is consistent with several studies showing that sensory properties of LFP and SUA at individual cortical sites are qualitatively different (Kreiman et al., 2006; Nielsen et al., 2006; Asher et al., 2007). Specifically, it has been suggested that stimulus-evoked LFP activity rather reflects the sensory characteristics of the intercortical input to an area that local processing within the region (Khawaja et al., 2009). Hence, VEP selectivity in V4 could reflect stimulus selectivity of regions pro- 
jecting to V4 rather than the stimulus selectivity generated within the area itself. The major feedforward input to V4 comes from thin stripe and interstripe regions in V2 (Zeki and Shipp, 1989; Xiao et al., 1999; Ungerleider and Pasternak, 2004), and especially thin stripe regions show high color-biased activity (Hubel and Livingstone, 1987; Roe and Ts'o, 1995; Shipp and Zeki, 2002; Gegenfurtner, 2003; Tootell et al., 2004; Xiao et al., 2003). Thin stripe regions project to specific subregions in $\mathrm{V} 4$, which would result in a non-uniform organization of color-sensitive LFP sites (Yoshioka et al., 1992; Shipp and Zeki, 1995; Xiao et al., 1999; Stepniewska et al., 2005). When we assessed the distribution of color sensitivity across individual LFP sites across the recording area, we observed that similarly tuned LFP sites are in closer vicinity to each other than less similarly tuned sites, resulting in a non-uniform distribution (supplemental Fig. 8, available at www.jneurosci.org as supplemental material). This observation is also consistent with the findings of Conway (2007) using fMRI and Zeki (1983c) using electrophysiology. Thus, our findings suggest that V4 receives inputs dominated by color signals and transforms them into multiple representations characterized by various spike-selectivity functions.

Finally, our results suggest that the degree to which visualevoked activity of the LFP is representative of the underlying neural processing at the single cell level may be brain-region specific. During identical stimulus conditions, we found differences in the relationship between visually evoked single unit activity and LFP responses in V4 and IFP that can only be attributed to factors that are unique to the area under study, at least within the context of the visual recognition task we used.

Our findings have thus implications for studies investigating neural correlates of visual cognition with electroencephalography. Whether mesoscopic signals, for example event-related potentials, correspond to dendritic input to a region, local processing within the region or spiking output from the region may depend on factors that are unique to the area under study, for example the topographic organization of neurons within the area and the distributions of input signals across cortical layers. Further work using laminar probes registering activity across all cortical layers is required to understand the details of this information flow. Another important factor likely influencing the relationship in sensory sensitivity between stimulus-evoked LFP responses and single neuron activity is the stimulus type. Almost all studies investigating the relationship between the signals used simple stimuli such as sparse noise and oriented gratings. In our study we used natural images, which are more characteristic of visual input in real world vision. Further studies comparing the effect of artificial and natural stimuli on spike and LFP tuning are necessary to elucidate the contribution of the stimulus type to the relationship between these signals. Ultimately, our study highlights the importance of studying both spiking activity as well as stimulus-evoked potentials for investigating their respective role in visual cognition.

\section{References}

Allison T, Begleiter A, McCarthy G, Roessler E, Nobre AC, Spencer DD (1993) Electrophysiological studies of color processing in human visual cortex. Electroencephalogr Clin Neurophysiol 88:343-355.

Asaad WF, Rainer G, Miller EK (2000) Task-specific neural activity in the primate prefrontal cortex. J Neurophysiol 84:451-459.

Asher I, Stark E, Abeles M, Prut Y (2007) Comparison of direction and object selectivity of local field potentials and single units in macaque posterior parietal cortex during prehension. J Neurophysiol 97:3684-3695.

Bartels A, Zeki S (2000) The architecture of the colour centre in the human visual brain: new results and a review. Eur J Neurosci 12:172-193.
Buchner H, Weyen U, Frackowiak RS, Romaya J, Zeki S (1994) The timing of visual evoked potential activity in human area V4. Proc Biol Sci 257:99-104.

Conway BR, Tsao DY (2006) Color architecture in alert macaque cortex revealed by FMRI. Cereb Cortex 16:1604-1613.

Conway BR, Tsao DY (2009) Color-tuned neurons are spatially clustered according to color preference within alert macaque posterior inferior temporal cortex. Proc Natl Acad Sci U S A 106:18034-18039.

Conway BR, Moeller S, Tsao DY (2007) Specialized color modules in macaque extrastriate cortex. Neuron 56:560-573.

Desimone R, Duncan J (1995) Neural mechanisms of selective visual attention. Annu Rev Neurosci 18:193-222.

Desimone R, Schein SJ (1987) Visual properties of neurons in area V4 of the macaque: sensitivity to stimulus form. J Neurophysiol 57:835-868.

Desimone R, Albright TD, Gross CG, Bruce C (1984) Stimulus-selective properties of inferior temporal neurons in the macaque. J Neurosci 4:2051-2062.

Duncan J (2001) An adaptive coding model of neural function in prefrontal cortex. Nat Rev Neurosci 2:820-829.

Felsen G, Dan Y (2005) A natural approach to studying vision. Nat Neurosci 8:1643-1646.

Freedman DJ, Riesenhuber M, Poggio T, Miller EK (2001) Categorical representation of visual stimuli in the primate prefrontal cortex. Science 291:312-316.

Fuster JM, Alexander GE (1971) Neuron activity related to short-term memory. Science 173, 652-654.

Gegenfurtner KR (2003) Cortical mechanisms of colour vision. Nat Rev Neurosci 4:563-572.

Gustavsen K, Gallant JL (2003) Shape perception: complex contour representation in visual area V4. Curr Biol 13:R234-R235.

Hillyard SA (1993) Electrical and magnetic brain recordings: contributions to cognitive neuroscience. Curr Opin Neurobiol 3:217-224.

Hubel DH, Livingstone MS (1987) Segregation of form, color, and stereopsis in primate area 18. J Neurosci 7:3378-3415.

Hubel DH, Wiesel TN (1968) Receptive fields and functional architecture of monkey striate cortex. J Physiol 195:215-243.

Katzner S, Nauhaus I, Benucci A, Bonin V, Ringach DL, Carandini M (2009) Local origin of field potentials in visual cortex. Neuron 61:35-41.

Khawaja FA, Tsui JM, Pack CC (2009) Pattern motion selectivity of spiking outputs and local field potentials in macaque visual cortex. J Neurosci 29:13702-13709.

Kobatake E, Tanaka K (1994) Neuronal selectivities to complex object features in the ventral visual pathway of the macaque cerebral cortex. J Neurophysiol 71:856-867.

Kreiman G, Hung CP, Kraskov A, Quiroga RQ, Poggio T, DiCarlo JJ (2006) Object selectivity of local field potentials and spikes in the macaque inferior temporal cortex. Neuron 49:433-445.

Kulikowski JJ, Robson AG, Murray IJ (2002) Scalp VEPs and intra-cortical responses to chromatic and achromatic stimuli in primates. Doc Ophthalmol 105:243-279.

Kusunoki M, Moutoussis K, Zeki S (2006) Effect of background colors on the tuning of color-selective cells in monkey area V4. J Neurophysiol 95:3047-3059.

Lee H, Simpson GV, Logothetis NK, Rainer G (2005) Phase locking of single neuron activity to theta oscillations during working memory in monkey extrastriate visual cortex. Neuron 45:147-156.

Leon MI, Shadlen MN (1999) Effect of expected reward magnitude on the response of neurons in the dorsolateral prefrontal cortex of the macaque. Neuron 24:415-425.

Liebe S, Fischer E, Logothetis N, Rainer G (2009) Color and shape interactions in the recognition of natural scenes by human and monkey observers. J Vis 9:1-16.

Liu J, Newsome WT (2006) Local field potential in cortical area MT: stimulus tuning and behavioral correlations. J Neurosci 26:7779-7790.

Lueck CJ, Zeki S, Friston KJ, Deiber MP, Cope P, Cunningham VJ, Lammertsma AA, Kennard C, Frackowiak RS (1989) The colour centre in the cerebral cortex of man. Nature 340:386-389.

Maunsell JH, Cook EP (2002) The role of attention in visual processing. Philos Trans R Soc Lond B Biol Sci 357:1063-1072.

Maunsell JH, Newsome WT (1987) Visual processing in monkey extrastriate cortex. Annu Rev Neurosci 10:363-401. 
Miller EK (2000) The prefrontal cortex and cognitive control. Nat Rev Neurosci 1:59-65.

Miller EK, Cohen JD (2001) An integrative theory of prefrontal cortex function. Annu Rev Neurosci 24:167-202.

Miller EK, Erickson CA, Desimone R (1996) Neural mechanisms of visual working memory in prefrontal cortex of the macaque. J Neurosci 16:5154-5167.

Mitzdorf U (1985) Current source-density method and application in cat cerebral cortex: investigation of evoked potentials and EEG phenomena. Physiol Rev 65:37-100.

Mitzdorf U (1994) Properties of cortical generators of event-related potentials. Pharmacopsychiatry 27:49-51.

Monosov IE, Trageser JC, Thompson KG (2008) Measurements of simultaneously recorded spiking activity and local field potentials suggest that spatial selection emerges in the frontal eye field. Neuron 57:614-625.

Murphey DK, Yoshor D, Beauchamp MS (2008) Perception matches selectivity in the human anterior color center. Curr Biol 18:216-220.

Nauhaus I, Benucci A, Carandini M, Ringach DL (2008) Neuronal selectivity and local map structure in visual cortex. Neuron 57:673-679.

Nielsen KJ, Logothetis NK, Rainer G (2006) Dissociation between local field potentials and spiking activity in macaque inferior temporal cortex reveals diagnosticity-based encoding of complex objects. J Neurosci 26:9639-9645.

Pasupathy A, Connor CE (2002) Population coding of shape in area V4. Nat Neurosci 5:1332-1338.

Rainer G (2008) Localizing cortical computations during visual selection. Neuron 57:480-481.

Rainer G, Miller EK (2000) Effects of visual experience on the representation of objects in the prefrontal cortex. Neuron 27:179-189.

Rainer G, Miller EK (2002) Timecourse of object-related neural activity in the primate prefrontal cortex during a short-term memory task. Eur J Neurosci 15:1244-1254.

Rainer G, Asaad WF, Miller EK (1998) Selective representation of relevant information by neurons in the primate prefrontal cortex. Nature 393:577-579.

Rainer G, Lee H, Logothetis NK (2004) The effect of learning on the function of monkey extrastriate visual cortex. PLoS Biol 2:E44.

Rao SC, Rainer G, Miller EK (1997) Integration of what and where in the primate prefrontal cortex. Science 276:821-824.

Roe AW, Ts'o DY (1995) Visual topography in primate V2: multiple representation across functional stripes. J Neurosci 15:3689-3715.

Schein SJ, Desimone R (1990) Spectral properties of V4 neurons in the macaque. J Neurosci 10:3369-3389.

Schein SJ, Marrocco RT, de Monasterio FM (1982) Is there a high concentration of color-selective cells in area V4 of monkey visual cortex? J Neurophysiol 47:193-213.

Schummers J, Mariño J, Sur M (2002) Synaptic integration by V1 neurons depends on location within the orientation map. Neuron 36:969-978.

Shipp S, Zeki S (2002) The functional organization of area V2, I: specialization across stripes and layers. Vis Neurosci 19:187-210.

Shipp, S, Zeki, S (1995) Segregation and convergence of specialised pathways in macaque monkey visual cortex. J Anat 187:547-562.

Stepniewska I, Collins CE, Kaas JH (2005) Reappraisal of DL/V4 boundaries based on connectivity patterns of dorsolateral visual cortex in macaques. Cereb Cortex 15:809-822.

Stoughton CM, Conway BR (2008) Neural basis for unique hues. Curr Biol 18:R698-R699.

Tanaka M, Weber H, Creutzfeldt OD (1986) Visual properties and spatial distribution of neurones in the visual association area on the prelunate gyrus of the awake monkey. Exp Brain Res 65:11-37.

Tanigawa H, Lu HD, Roe AW (2010) Functional organization for color and orientation in macaque V4. Nat Neurosci 13:1542-1548.

Tootell RB, Nelissen K, Vanduffel W, Orban GA (2004) Search for color 'center(s)' in macaque visual cortex. Cereb Cortex 14:353-363.

Treue S, Maunsell JH (1996) Attentional modulation of visual motion processing in cortical areas MT and MST. Nature 382:539-541.

Ungerleider, LG, Pasternak, T (2004) Ventral and dorsal processing streams. In: The visual neurosciences (Chalupa LM, Werner JS, eds), pp. 541-562. Cambridge, MA: MIT.

Victor JD, Purpura K, Katz E, Mao B (1994) Population encoding of spatial frequency, orientation, and color in macaque V1. J Neurophysiol 72:2151-2166.

Wallis JD (2007) Neuronal mechanisms in prefrontal cortex underlying adaptive choice behavior. Ann N Y Acad Sci 1121:447-460.

Wallis JD, Miller EK (2003) Neuronal activity in primate dorsolateral and orbital prefrontal cortex during performance of a reward preference task. Eur J Neurosci 18:2069-2081.

Walsh V, Carden D, Butler SR, Kulikowski JJ (1993) The effects of V4 lesions on the visual abilities of macaques: hue discrimination and colour constancy. Behav Brain Res 53:51-62.

Wichmann FA, Hill NJ (2001a) The psychometric function: I. Fitting, sampling, and goodness of fit. Percept Psychophys 63:1293-1313.

Wichmann FA, Hill NJ (2001b) The psychometric function: II. Bootstrapbased confidence intervals and sampling. Percept Psychophys 63:1314-1329.

Wild HM, Butler SR, Carden, D. Kulikowski J (1985) Primate cortical area V4 important for colour constancy but not wavelength discrimination. Nature 313:133-135.

Xiao Y, Zych A, Felleman DJ (1999) Segregation and convergence of functionally defined V2 thin stripe and interstripe compartment projections to area V4 of macaques. Cereb Cortex 9:792-804.

Xiao Y, Wang Y, Felleman DJ (2003) A spatially organized representation of colour in macaque cortical area V2. Nature 421:535-539.

Xing D, Yeh CI, Shapley RM (2009) Spatial spread of the local field potential and its laminar variation in visual cortex. J Neurosci 29:11540-11549.

Yajeya J, Quintana J, Fuster JM (1988) Prefrontal representation of stimulus attributes during delay tasks. II. The role of behavioral significance. Brain Res 474:222-230.

Yoshioka T, Levitt JB, Lund JS (1992) Intrinsic lattice connections of macaque monkey visual cortical area V4. J Neurosci 12:2785-2802.

Zaksas D, Pasternak T (2006) Directional signals in the prefrontal cortex and in area MT during a working memory for visual motion task. J Neurosci 26:11726-11742.

Zeki S (1980) The representation of colours in the cerebral cortex. Nature 284:412-418.

Zeki S (1983a) Colour coding in the cerebral cortex: the reaction of cells in monkey visual cortex to wavelengths and colours. Neuroscience 9:741-765.

Zeki S (1983b) Colour coding in the cerebral cortex: the responses of wavelength-selective and colour-coded cells in monkey visual cortex to changes in wavelength composition. Neuroscience 9:767-781.

Zeki S (1983c) The distribution of wavelength and orientation selective cells in different areas of monkey visual cortex. Proc R Soc Lond B Biol Sci 217:449-470.

Zeki S, Shipp S (1989) Modular connections between areas V2 and V4 of macaque monkey visual cortex. Eur J Neurosci 1:494-506.

Zeki SM (1973) Colour coding in rhesus monkey prestriate cortex. Brain Res 53:422-427. 